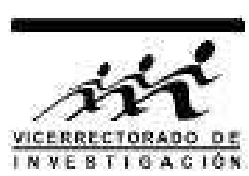

\title{
El átomo muónico de Bohr
}

\author{
Jorge A. Bravo*1 \\ ${ }^{1}$ Facultad de Ciencias Físicas, Universidad Nacional Mayor de San Marcos, Lima, Perú
}

Recibido 19 noviembre 2016 - Aceptado 15 diciembre 2016

\begin{abstract}
La teoría que Bohr aplicó en 1913 para describir los orbitales electrónicos se extiende al caso de los orbitales de muones en un átomo muónico. Cabe la posibilidad que para nucleídos con número atómico suficientemente grande, estas órbitas ocupen el volumen nuclear y, por lo tanto, el modelo de núcleo puntual que Bohr utilizó ya no es aplicable. Este estudio deduce que para nucleídos con $Z \geq 46$ el radio de la primera órbita de Bohr yace dentro del volumen nuclear; asimismo, se deduce que las órbitas muónicas de orden mayor yacen fuera del volumen nuclear.
\end{abstract}

Palabras claves: Modelo de Bohr, muones, átomos muónicos.

\section{The muonic atom of Bohr}

The theory that Bohr applied in 1913 to describe the electronic orbitals is extended to the muonic orbitals in a muonic atom. There is a possibility that for nuclides with sufficiently large atomic number, these orbitals occupy the nuclear volume and, therefore, the puntual nuclear model that Bohr utilized is not applicable. In this study, we deduce that for nuclides with $Z \geq 46$ the Bohr radius of the first level stay inner of nuclear volume; also, we deduce that the muonic orbitals with bigger order stay out of nuclear volume.

Keywords: Bohr model, muons, muonic atoms.

En este trabajo se presenta un modelo físico que permite deducir las ecuaciones que satisfacen los orbitales de un átomo muónico en el marco del formalismo utilizado por Niels Bohr 1 en 1913 para describir orbitales electrónicos para lo cual el modelo de núcleo puntual fue adecuado. El muón fue descubierto en 1936 por Carl Anderson y Seth Neddermeyer 2]. Estas partículas asemejan electrones con una masa 206.768 veces mayor y, por lo tanto, sus órbitas poseen radios mucho más pequeños en la misma proporción en el caso del átomo del hidrógeno. Pero para átomos con número atómico suficientemente grande cabe la posibilidad que los radios de Bohr correspondientes sean menores que los radios nucleares respectivos, de manera que el modelo de núcleo puntual deja de ser aceptable. En estos casos es necesario modificar la forma analítica del potencial de Coulomb dentro del volumen nuclear. Los resultados de este trabajo son importantes para fines de la enseñanza de las diferentes escalas en tamaño y energía entre los orbitales electrónicos y muónicos. Asimismo, son importantes como referencia en el cálculo de estos mismos orbitales haciendo uso de la teoría cuántica relativística.

Utilizaremos unidades cgs. Consideramos una distribución uniforme de carga eléctrica dentro del volumen nuclear con una densidad que viene dada por

$$
\rho(r)=\left\{\begin{array}{cc}
\rho_{0}, & 0<r<R_{n} \\
0, & r>R_{n}
\end{array}\right.
$$

donde $\rho_{0}$ y $R_{n}$ son la densidad constante y el radio nuclear, respectivamente, de modo tal que $Z e=\rho_{0} V_{n}$, siendo $V_{n}$ el volumen nuclear expresado por

$$
V_{n}=\frac{4 \pi}{3} R_{n}^{3}
$$

Esta distribución de carga nuclear da lugar al siguiente potencial de Coulomb dentro y fuera del núcleo

$$
V(r)=\left\{\begin{array}{cc}
\frac{3 e Z}{2 R_{n}}\left(1-\frac{r^{2}}{3 R_{n}^{2}}\right) & 0 \leq r<R_{n} \\
\frac{e Z}{r} & r>R_{n} .
\end{array}\right.
$$

De estas expresiones podemos deducir el campo eléctrico aplicando el operador del gradiente

$$
E(r)=\left\{\begin{array}{cc}
\frac{e Z r}{R_{n}^{3}} & 0 \leq r<R_{n} \\
\frac{e Z}{r^{2}} & r>R_{n}
\end{array},\right.
$$

${ }^{*}$ jbravoc@unmsm.edu.pe 
donde $E(r)$ es la componente radial del campo eléctrico; las otras componentes se anulan.

Ahora consideramos las condiciones de conservación de momento lineal y energía total para órbitas muónicas circulares, dados por

$$
\begin{aligned}
-\frac{\mu v^{2}}{r} & =-e E(r) \quad \text { y } \\
\frac{\mu v^{2}}{2}-e V(r) & =E_{t},
\end{aligned}
$$

respectivamente, donde $\mu$ es la masa reducida y $-e$ es la carga del muón y $E_{t}$ es la energía total del muón. La forma explícita de $E(r)$ depende de si $r>R_{n}$ ó $r<R_{n}$. De manera particular nos interesa el caso en que $r<R_{n}$.

A continuación consideramos la condición de cuantización del momento angular orbital que fuera introducida por Niels Bohr

$$
r \mu v=n \hbar, \quad n=\text { entero }>0 .
$$

Con la ayuda de la Ec.(7) despejamos $v$ en función de $r$, y sustituimos en la Ec.(5) para obtener una expresión para $r<R n$ dao por

$$
r^{4}=\frac{n^{2}}{Z} a_{\mu} R_{n}^{3} \quad \text { ó } \quad \frac{r_{n}}{R_{n}}=\left(n^{2} \frac{a_{\mu}}{Z R_{n}}\right)^{1 / 4},
$$

donde $a_{\mu}=\left(m_{e} / m_{\mu}\right) a_{B}$ es el radio muónico de Bohr en términos de la masa el electrón $m_{e}$ y de la masa reducida del muón $m_{\mu}$, y del radio de Bohr que viene dado por

$$
a_{B}=\frac{\hbar^{2}}{e^{2} m_{e}} .
$$

Es importante notar que en el caso límite en que $r_{n}=R_{n}$ se cumple la relación $a_{\mu}=Z R_{n}$.

Para el caso en que $r>R_{n}$ tenemos el siguiente resultado

$$
r_{n}=n^{2} \frac{a_{\mu}}{Z}, \quad n=\text { entero }>0 .
$$

A continuación se deduce la energía total del muón utilizando la Ec.(6),

$$
E_{t_{n}}=\left\{\begin{array}{cl}
\frac{1}{2} \frac{Z e^{2}}{R_{n}}\left(\frac{n^{2} a_{\mu} R_{n}}{Z r_{n}^{2}}-3+\frac{r_{n}^{2}}{R_{n}^{2}}\right), & r_{n}<R_{n} . \\
-\frac{1}{2} \frac{Z^{2} e^{2}}{n^{2} a_{\mu}}, & r_{n}>R_{n} .
\end{array}\right.
$$

Se puede verificar que estas expresiones satisfacen la continuidad cuando $r_{n}=R_{n}$ para $n=1$, por cuanto en este caso $Z R_{n}=a_{\mu}$; en este caso no tiene sentido considerar otros valores de $n$ pues si esta condición se cumple, para $n>1$, es decir, $r_{n}>R_{n}$ hay que usar la Ec.(11b).

Utilizando la Ec.(8) se puede explorar la posibilidad que $r_{n}=R_{n}$ cuando $n=1$, que corresponde a la órbita muónica más interna. Esta condición es equivalente a imponer que $Z R_{n}=a_{\mu}$. Para lograr nuestro objetivo es necesario tener una relación entre $R_{n}$ y $Z$. Para este fin utilizamos la relación $R_{n}=1.2 \times A^{1 / 3} F$ y tanteamos $A=2.3 Z$ que se cumple de manera aproximada para nucleídos estables de $Z \approx 50$.

Obtenemos la siguiente expresión

$$
1.2 Z(2.3 Z)^{1 / 3} F=a_{\mu}=256 F \Rightarrow Z^{4 / 3}=161.6
$$

Que tiene como solución $Z=45.3$.

Este resultado garantiza que a partir de $Z=46$ puedan ocurrir orbitales muónicos con $r_{1}<R_{n}$. Para verificar esta premisa consideramos nucleídos en esta vecindad y los resultados se dan en la Tabla 1.

\begin{tabular}{cccccc}
\hline Elemento & $Z$ & $A$ & $r_{1} / R_{n}$ & $\begin{array}{c}E_{t 1} \\
(\mathrm{MeV})\end{array}$ & $\begin{array}{c}E_{t 1 p} \\
(\mathrm{MeV})\end{array}$ \\
\hline $\mathrm{Pd}$ & 46 & 106 & 0.995 & -5.95 & -5.96 \\
$\mathrm{Ag}$ & 47 & 110 & 0.987 & -6.20 & -6.22 \\
$\mathrm{Ba}$ & 56 & 138 & 0.927 & -8.34 & -8.83 \\
$\mathrm{~W}$ & 74 & 186 & 0.843 & 12.27 & -15.42 \\
$\mathrm{~Pb}$ & 82 & 208 & 0.814 & -13.90 & -18.93 \\
$\mathrm{U}$ & 92 & 238 & 0.782 & -15.82 & -23.73 \\
$\mathrm{Cf}$ & 98 & 252 & 0.766 & -16.97 & -27.04 \\
\hline
\end{tabular}

Tabla 1: Valores del radio y energía de la primera órbita muónica de Bohr para un conjunto de nucleídos. Se incluye la energía del modelo puntual.

Para el caso de la segunda órbita de Bohr aplicamos nuevamente la Ec.(8) con $n=2$ y la relación $A=2.6 Z$, que es aparente para nucleídos pesados. En este caso la Ec.(12) deviene en $Z^{4 / 3}=520.6$, lo cual resultaa $Z=124$, que corresponde a nucleídos superpesados que todavía no han sido sintetizados. De manera general, tenemos que $r_{2}>R_{n}$. Para estos casos y otras órbitas de mayor orden, la fórmula de la Ec.(11b) es aplicable. Como se puede observar, a medida que aumenta el número atómico del nucleído la discrepancia con los valores del modelo puntual se hacen más notorios.

En conclusión, se ha cumplido con extender la teoría de Bohr al estudio de los átomos muónicos. Es interesante observar que sólo la primera órbita de Bohr puede ocupar el volumen nuclear de los nucleídos existentes y esto ocurre para nucleídos con $Z \geq 46$. Asimismo, es interesante notar la magnitud de las energías de estos orbitales que superan en varios órdenes de magnitud las energías de los orbitales electrónicos.

Este modelo es, sin embargo, sólo una primera aproximación. En la actualidad, en esta Facultad, se está elaborando un programa que calcula las funciones de onda de estos átomos muónicos que toma en cuenta efectos cuánticos y relativísticos. $Y$ será interesante poder contrastar e interpretar las diferencias en los resultados.

En la actualidad el estudio de los átomos muónicos es motivo de especial atención en varios laboratorios del planeta y merece ser un tema de enseñanza en los cursos de Física Moderna de la actualidad. 
Agradezco a la Escuela Académico Profesional de Física de esta Facultad por haberme encargado el dictado del curso de Física Nuclear cuyo cumplimiento me ha permiti- do desarrollar varias ideas que han contribuido a la mejor enseñanza de este curso y al mejor entendimiento de la Física.

\section{Referencias}

[1] N. Bohr; On the constitution of atoms and molecules, Philos. Mag. 26, 1-24 (1913).

[2] Carl D. Anderson and Seth Neddermeyer; Cloud chamber observations of cosmic rays at 4300 meters and near sea level, Phys. Rev. 50, 263-271 (1936).

[3] Harald A. Enge; Introduction to Nuclear Physics; Addison-Wesley, Co., New York (1966). 